\title{
Action de la flore microbienne du tractus digestif et de l'aliment sur l'absorption du taurocholate de sodium chez le rat
}

\author{
par M. RIOTTOT, E. SACQUET, C. LEPRINCE, C. MEJEAN \\ Laboratoire des Animaux sans Germes du C.N.R.S., \\ I.N.R.A., 78350 Jouy-en-Josas, France.
}

Summary. Effects of intestinal microflora and dief on sodium taurocholate absorption in the rat.

The ileal absorption of sodium taurocholate (TCNa) depends on different factors which have not been well established. In the axenic rat fed a semi-purified diet, the rate of the gastrointestinal transit was lower (mechanical factor) and the ileal absorption rate of TCNa determined in the anaesthetized animal after perfusion was higher (unknown factor) than in the conventional rat. Both factors led to a slow down of the TCNa enterohepatic cycle and to an increase of the pool size of this bile salt in the small intestine.

As compared to a semi-purified diet, a standard dief increased the TCNa absorption rate measured by perfusion in vivo in axenic and conventional rats. The intestinal pool size of TCNa was increased in both groups of animals.

When lactose was introduced into the semi-purified diet, TCNa absorption increased in the conventional rat. When lactose was introduced into drinking water, the absorption was increased, but in a different way.

La quantité de sels biliaires présente au niveau de l'intestin grêle du rał varie en fonction de la flore microbienne et du régime alimentaire (tabl. 1). Elle est plus élevée chez le rat axénique que chez le rat holoxénique. Elle est plus élevée chez les rats qui

\section{TABLEAU 1}

Quantité de sels biliaires exprimée en micromoles par $100 \mathrm{~g}$ de poids corporel au niveau de l'intestin grêle de rats axéniques et holoxéniques qui reçoivent deux aliments différents

(Moyenne \pm écart-type de la moyenne de 4 rats)

\begin{tabular}{ccc}
\hline Etat microbien & Aliment DC * & Aliment DP* \\
\hline Axénique $\ldots \ldots \ldots \ldots \ldots$ & $73,4 \pm 3,1$ & $105 \pm 5,5$ \\
\hline Holoxénique $\ldots \ldots \ldots \ldots$ & $21,1 \pm 1,0$ & $50,8 \pm 5,3$ \\
\hline
\end{tabular}

* Composition des aliments : voir tableau 2. 
reçoivent un aliment commercial de composition complexe, DP, que chez ceux qui reçoivent un aliment semi-synthétique, DC, (tabl. 2). Cette élévation est d'un même ordre de grandeur chez le rat axénique et chez le rat holoxénique : elle est donc indépendante de la flore microbienne.

TABLEAU 2

Composition des régimes alimentaires $D, D C, D P$ (p. 100 en poids)

\begin{tabular}{|c|c|c|c|}
\hline$D$ & & $D C$ & DP \\
\hline \multirow[t]{2}{*}{$\begin{array}{l}\text { Caséine } \\
\text { Amidon de Maïs } \\
\text { Huile de Maïs } \\
\text { Sels minéraux } \\
\text { Cellulose }\end{array}$} & $\begin{array}{l}: 22 \\
: 58 \\
: 9 \\
: \quad 4,5 \\
: \quad 5\end{array}$ & $\begin{array}{l}\text { Identique } \\
\text { à } D\end{array}$ & $\begin{array}{l}\text { Maïs, Blé, Farine basse de blé, Issues de } \\
\text { blé, Tourteaux de Soja, Arachides, Farine } \\
\text { de Poisson, Levure, Graisses animales, } \\
\text { Mélasse, Lactosérum, Farine de Luzerne } \\
\text { déshydratée, Composé minéral. }\end{array}$ \\
\hline & & & $\begin{array}{l}\text { Matières protéiques brutes, min. } 21 \text { p. } 100 . \\
\text { Matières grasses, min. } 3,5 \text { p. } 100 \\
\text { Matières cellulosiques, max. } 5 \text { p. } 100\end{array}$ \\
\hline Cholestérol & $: 0$ & 0,09 & 0,09 \\
\hline
\end{tabular}

Ces régimes sont stérilisés par autoclavage à $120^{\circ} \mathrm{C}$ pendant $20 \mathrm{mn}$.

Ces valeurs différentes du pool de l'intestin grêle peuvent avoir comme origine des différences de vitesse du transit intestinal. C'est très vraisemblablement le cas chez le rat axénique qui a un transit intestinal plus lent que le rat holoxénique (Sacquet, Garnier et Raibaud, 1970). Mais il est également possible que ces différences relèvent de variations de la vitesse d'absorption des sels biliaires au niveau de l'iléon. Les sels biliaires sont principalement absorbés au niveau de cette région (Dietschy, Salomon et Siperstein, 1966). Une absorption plus rapide des sels biliaires dans cette région a pour effet un retour en quantité plus importante des sels biliaires dans la région antérieure de l'intestin grêle, via le système porte, le foie et les canaux biliaires. Les sels biliaires sont ainsi mieux retenus au niveau du cycle enférohépatique et donc au niveau de l'intestin grêle puisque la plus grande partie de ce cycle se déroule au niveau de cet organe.

Pour vérifier cette hypothèse, des rats axéniques et holoxéniques soumis soit au régime $D$, soit au régime DP ont fait l'objet de perfusion par du (4-14C)-taurocholate de sodium, selon une technique précédemment décrite (Riottot et al., 1975).

Le tableau 3 montre que les rats qui ingèrent le régime DP ont une vitesse d'absorption iléale du taurocholate de sodium plus grande que ceux qui reçoivent le régime $D$. Chez les rats qui reçoivent le régime $D$, une différence significative peut être mise en évidence entre axéniques et holoxéniques, alors que cela n'est pas possible chez les rats qui reçoivent le régime DP. 
TABLEAU 3

Vifesse d'absorption iléale du taurocholate de sodium ( $\mathrm{TCNa}$ ) en nanomoles par mn et par cm d'intestin (Moyenne \pm écart-type de la moyenne de 6 rats)

\begin{tabular}{|c|c|c|c|}
\hline Etat microbien & $\begin{array}{l}\text { Concentration du } \\
\text { perfusat en } \mathrm{TCNa}\end{array}$ & Aliment D & Aliment DP \\
\hline Axénique $\ldots \ldots \ldots \ldots \ldots$ & $\begin{array}{l}15 \mathrm{mM} \\
25 \mathrm{mM}\end{array}$ & $\begin{array}{l}4,8 \pm 1,0 a^{*} \\
8,5 \pm 0,9 b\end{array}$ & $\begin{array}{r}7,6 \pm 1,1 b \\
17,7 \pm 2,4 c\end{array}$ \\
\hline Holoxénique & $\begin{array}{l}15 \mathrm{mM} \\
25 \mathrm{mM}\end{array}$ & $\begin{array}{l}3,4 \pm 0,7 a \\
4,5 \pm 0,5 a\end{array}$ & $\begin{array}{r}7,5 \pm 0,9 b \\
14,4 \pm 2,2 c\end{array}$ \\
\hline
\end{tabular}

* Les lettres désignent les valeurs qui ne sont pas différentes : $p>0,05$.

Cette expérience conduit à s'interroger sur la nature des facteurs alimentaires qui déterminent cet accroissement de la vitesse d'absorption du taurocholate de sodium Le tableau 4 représente les vitesses d'absorption du (4-14C)-taurocholate de sodium chez des rats holoxéniques qui reçoivent du lactose : les rats DLA sont nourris à l'aide de l'aliment semi-synthétique $D$ qui reçoit 10 p. 100 en poids de lactose avant d'être autoclavé ; les rats DLB ne reçoivent pas de lactose dans leur aliment $D$, mais

\section{TABLEAU 4}

Vitesse d'absorption iléale du faurocholate de sodium (TCNa) en nanomoles par mn par cm d'intestin (Moyenne \pm écart-type de la moyenne de 5 rats)

\begin{tabular}{cccc}
\hline $\begin{array}{c}\text { Concentration du } \\
\text { perfusat en TCNa }\end{array}$ & Lot D & Lot DLA & Lot DLB \\
\hline $5 \mathrm{mM}$ & $4,9 \pm 0,6 \mathrm{a}^{*}$ & $8,0 \pm 1,0 \mathrm{~b}$ & $5,9 \pm 1,0 \mathrm{a}$ \\
\hline $15 \mathrm{mM}$ & $3,4 \pm 0,7 \mathrm{a}$ & $8,5 \pm 0,9 \mathrm{~b}$ & $11,5 \pm 1,2 \mathrm{c}$ \\
\hline $25 \mathrm{mM}$ & $4,5 \pm 0,5 \mathrm{a}$ & $16,9 \pm 1,9$ & $11,6 \pm 1,7 \mathrm{c}$ \\
\hline
\end{tabular}

* Les lettres désignent les valeurs qui ne diffèrent pas significativement au risque $5 p .100(p>0,05)$

dans l'eau de boisson et ingèrent de cette manière une quantité de lactose sensiblement égale à celle que l'aliment apporte aux rats DLA. Chez ces deux groupes de rats, la vitesse d'absorption du taurocholate de sodium est plus grande que chez les rats $D$, qui ne reçoivent pas de lactose. Chez les rats $D L A$, la quantité de sels biliaires au niveau de l'intestin grêle est aussi plus élevée que chez les rats $D(P=0,05)$ (tabl. 5). L'excrétion fécale et donc la synthèse des acides biliaires n'est pas significativement plus grande chez les rats DLA : l'accroissement du pool des sels biliaires au 
niveau de l'intestin grêle, l'accroissement de la vitesse d'absorption iléale du taurocholate de sodium et vraisemblablement de l'ensemble des sels biliaires, n'est donc pas un phénomène secondaire à une augmentation de la synthèse des sels biliaires sous l'action du lactose.

\section{TABLEAU 5}

Excrétion fécale journalière ef quantifé de sels bilicires totaux au niveau de l'intestin grêle en micromoles par $100 \mathrm{~g}$ de poids corporel

(Moyenne \pm écart-type de la moyenne de 4 rats)

\begin{tabular}{ccc}
\hline Lots & $\mathrm{D}$ & DLA \\
\hline Intestin grêle $\ldots \ldots \ldots \ldots$ & $22,7 \pm 1,2$ & $33,2 \pm 4,1$ \\
\hline Excrétion fécale........ & $12,9 \pm 0,9$ & $13,7 \pm 0,9$ \\
\hline
\end{tabular}

Enfin le régime DLA contient beaucoup plus de lactose que l'aliment DP qui cependant accroît la vitesse d'absorption du taurocholate de sodium. Ceci peut s'expliquer de deux manières : ou bien le lactose est actif à des concentrations beaucoup plus faibles que celle qu'il présente dans l'aliment DLA ; ou bien il existe dans l'aliment DP d'autres facteurs que le lactose qui modifient cette vitesse d'absorption.

Commission CNERMA Digestion-Absorption, Tours, 13 novembre 1976.

\section{Références}

DIETSCHY J. M., SALOMON H. S., SIPERSTEIN M. D., 1966. Bile acid metabolism I. Studies on the mechanisms of intestinal transport. J. clin. Invest., 45, 832-846.

RIOTTOT M., SACQUET E., MEJEAN C., LEPRINCE C., 1975. Effect of diet and microbial flora on intestinal absorption of sodium taurocholate in germ-free and conventional rats. Nutr. Rep. Int., 12, 1-7.

SACQUET E., GARNIER H., RAIBAUD P., 1970. Etude de la vitesse du transit gastro-intestinal des spores d'une souche thermophile stricte de Bacillus subtilis chez le rat holoxénique, le rat axénique, le rat axénique caecectomisé. C. R. Soc. Biol., 164, 352. 